\title{
Evidências de condutas fisioterapêuticas na prevenção de quedas em idosos: uma
} revisão de literatura integrativa

\author{
Evidence of physiotherapeutic behavior in the prevention of falls in the elderly: an integrative \\ literature review
}

Evidencia de conductas fisioterapéuticas e la prevención de caídas en ancianos: revisión integradora de la literatura

Tatiany Cristina Viana Santos ORCID: https://orcid.org/0000-0003-0681-7217 Faculdade do Vale do Jaguaribe, Brasil E-mail: tatianycristina94@gmail.com

Roque Ribeiro da Silva Júnior ORCID: https://orcid.org/0000-0001-9517-6823 Universidade do Estado do Rio Grande do Norte, Brasil E-mail: roquejunior@alu.uern.br

Claudia Vaz Pupo de Mello

ORCID: https://orcid.org/0000-0002-7091-9114 Faculdade do Vale do Jaguaribe, Brasil E-mail: claudia.pupo@fvj.br

\begin{abstract}
Resumo
Objetivo: o presente estudo visa traçar o perfil epidemiológico dos pacientes atendidos no setor de fisioterapia de uma clínica- Introdução: Envelhecer faz parte de um processo natural e fisiológico, contudo, a pessoa idosa também fica mais propensa a cair devido as alterações funcionais, visuais e auditivas, o que aumenta também o risco de morte, visto que a queda é o acidente mais comum entre idosos. Sendo assim, o tratamento fisioterapêutico é muito importante pois, através de uma avaliação, podem ser formados protocolos que visam a prevenção de quedas. Objetivo: Buscar na literatura as melhores evidências para prevenção de quedas em idosos. Metodologia: Refere-se à uma revisão de literatura integrativa em que foi feito um levantamento na bibliografia sobre as abordagens fisioterapêuticas que apresentam melhores evidências para prevenção de quedas em idosos. As bases de dados utilizadas foram PubMed, PEDro e Cochrane na busca de ensaios clínicos randomizados com importância científica dos últimos cinco anos (2016-2021). Resultados: Foram achados 345 artigos, que, após passarem pelos critérios de inclusão, exclusão e as duas etapas de avaliação, restou um total 5 ensaios clínicos randomizados que foram colocados à disposição de acordo com autores, título, ano de publicação, objetivo geral e principais considerações. Considerações Finais: O estudo revelou que programas de exercícios físico e terapia com sistema de posturografia apresentaram melhores resultados para prevenção e diminuição do risco de quedas em idosos.
\end{abstract}

Palavras-chave: Idoso; Prevenção de quedas; Fisioterapia; Tratamento.

\begin{abstract}
Objective: this study aims to trace the epidemiological profile of patients seen in the physiotherapy sector of a clinic Introduction: Aging is part of a natural and physiological process, however, the elderly is also more likely to fall due to functional and visual changes and hearing, which also increases the risk of death, since falling is the most common accident among the elderly. Therefore, physical therapy treatment is very important because, through an assessment, protocols can be created to prevent falls. Objective: Search the literature for the best evidence for preventing falls in the elderly. Methodology: Refers to an integrative literature review in which a survey was conducted in the bibliography on physical therapy approaches that present the best evidence for the prevention of falls in the elderly. The databases used were PubMed, PEDro and Cochrane in the search for randomized clinical trials with scientific importance in the last five years (2016-2021). Results: A total of 345 articles were found, which, after passing the inclusion, exclusion and two stages of evaluation, left a total of 5 randomized clinical trials that were made available according to authors, title, year of publication, general objective, and main considerations. Final Considerations: The study revealed that physical exercise programs and therapy with a posturography system had better results for preventing and reducing the risk of falls in the elderly.
\end{abstract}

Keywords: Old man; Fall prevention; Physiotherapy; Treatment. 


\begin{abstract}
Resumen
Objetivo: este estudio tiene como objetivo rastrear el perfil epidemiológico de los pacientes atendidos en el sector de fisioterapia de una clínica - Introducción: El envejecimiento es parte de un proceso natural y fisiológico, sin embargo, los ancianos también tienen más probabilidades de caer debido a cambios funcionales y visuales y audición, lo que también aumenta el riesgo de muerte, ya que las caídas son el accidente más común entre los ancianos. Por tanto, el tratamiento de fisioterapia es muy importante porque, a través de una valoración, se pueden crear protocolos para prevenir caídas. Objetivo: Buscar en la literatura la mejor evidencia para prevenir caídas en ancianos. Metodología: Se refiere a una revisión integradora de la literatura en la que se realizó una encuesta en la bibliografía sobre los enfoques de fisioterapia que presentan la mejor evidencia para la prevención de caídas en el anciano. Las bases de datos utilizadas fueron PubMed, PEDro y Cochrane en la búsqueda de ensayos clínicos aleatorizados con relevancia científica en los últimos cinco años (2016-2021). Resultados: Se encontraron un total de 345 artículos, los cuales, luego de pasar la inclusión, exclusión y dos etapas de evaluación, dejaron un total de 5 ensayos clínicos aleatorizados que se pusieron a disposición según autores, título, año de publicación, objetivo general y principal. consideraciones. Consideraciones finales: El estudio reveló que los programas de ejercicio físico y la terapia con sistema de posturografía tuvieron mejores resultados para prevenir y reducir el riesgo de caídas en los ancianos.
\end{abstract}

Palabras clave: Anciano; Prevención de caídas; Fisioterapia; Tratamiento.

\title{
1. Introdução
}

O Estatuto do Idoso, Lei $\mathrm{n}^{\circ} 10.741$ de $1^{\circ}$ de outubro de 2003, define como idoso a pessoa com 60 anos ou mais. (Brasil, 2003). Envelhecer é um processo natural, fisiológico, progressivo e não patológico, onde há diminuição progressiva da reserva funcional dos indivíduos(senescência) o que, em condições normais, não provoca qualquer problema relacionado à saúde. (Xavier et al,2019).

Precisamente, no Brasil, a população de idosos tem aumentado cada vez mais. Calcula-se que o país terá mais e 40,7 milhões de pessoas com idade acima de 65 anos em 2030. (Xavier \& Pedro,2019) Esse aumento acontece porque a taxa de fecundidade diminuiu, e prevaleceu uma maior quantidade de idosos e gerando maior exigência dos serviços de saúde para essa população. (Oliveira, 2019).

Nesse cenário, devido ao processo de envelhecimento o risco de acidentes se torna alto, pois ocorrem mudanças na percepção visual e auditiva, diminuição da massa muscular, óssea e força (Phelan \& Ritchey, 2018). De maneira que, o tempo o torne o indivíduo menos capaz de fazer frente ao estresse do meio ambiente e, portanto, aumente a possibilidade de morte (Lopes et. al,2019; Lusardi et al.,2017).

Além disso, dentre os acidentes que possam causar lesões, modificar a capacidade de função e mesmo gerar a morte nos idosos, o mais comum é a queda (Chittrakul et al., 2020). A queda é entendida como um evento acidental não intencional que resulta na mudança de posição do indivíduo para mais próximo do solo, em relação a sua posição inicial. (Silva et al., 2017; Lipardo \& Tsang, 2018).

Segundo Sherrington et al (2017) tratando de saúde, os fatores de risco são probabilidades aumentadas de agravo à saúde do indivíduo. Os fatores de risco de quedas são consequências que ocorrem por alterações internas e externas; sendo internas as alterações fisiológicas relacionadas ao envelhecimento, de doenças e/ou efeitos causados pelos medicamentos. (Zhang et al.,2019). Destacando a dificuldade na marcha, a redução de força muscular, a restrição da ADM, dificuldade de equilíbrio, de visão, de cognição e a falta de exercício físico; e externos os que dependem de circunstâncias sociais e ambientais que geram dificuldade ao idoso. (Souza et al., 2017; Park, 2018).

Desta forma, se antecipar para tais situações é importante uma vez que com ações preventivas pode-se impedir a ocorrência destes acidentes (Morello et al.,2019). O fisioterapeuta é de grande importância na prevenção de quedas em idosos, sendo assim, capaz de fazer um apanhado da realidade do idoso, e, em seguida, determinar programas aplicáveis aos casos clínicos com efeito específico sobre a prevenção de quedas (Costa et. al, 2021; Lach \& Noimontree, 2018). 
Sendo assim, no tratamento, o fisioterapeuta após uma minuciosa avaliação, é apto a planejar um programa eficiente para à prevenção de quedas, através da realização de protocolos onde é possível ser treinado força muscular e equilíbrio do idoso. (Costa et. al, 2021; Liu-Ambrose et al.,2019).

Diante do que foi exposto o seguinte trabalho será destinado a responder a seguinte pergunta PICOT: P- idosos sem alteração no sistema locomotor (a partir de 60 anos) I- técnicas de fisioterapia C- comparado a nada O- redução de quedas Tensaios clínicos.

Portanto, o objetivo da pesquisa foi realizar um levantamento na literatura sobre as abordagens fisioterapêuticas na prevenção de quedas em idosos que apresentem melhores evidências.

\section{Metodologia}

Este estudo trata-se de uma revisão bibliográfica integrativa através de um levantamento na literatura sobre as abordagens fisioterapêuticas na prevenção de quedas em idosos que apresente melhores evidências. Dessa forma, a revisão bibliográfica integrativa objetiva resumir resultados de forma organizada, conclusa e extensa, possibilitando informações ampliadas sobre o assunto. (Ercole et al., 2014).

A revisão bibliográfica integrativa vai apresentar um entendimento mais amplo do conteúdo através de uma investigação de pesquisas passadas sobre um tema em específico, contendo fundamento diante da compilação de estudos que já foram publicados, de caráter metodológico que pode mudar ou não, sendo experimental ou não experimental, combinado a literatura baseada na experiência e na observação, que contribui para o desenvolvimento de estudos científicos. (Botelho et al., 2011).

A pesquisa foi realizada entre os meses de setembro de 2021 à outubro de 2021, nas bases de dados PubMed, Physiotherapy Evidence Database (PEDro) e Cochrane.

Os descritores foram selecionados de acordo com o Medical Subject Headings (MeSH), que são: Old Age, Falls, Physical Therapy. Para pesquisas em base de dados de predominância de língua inglesa, como PubMed, PEDro e Cochrane, foram utilizados o operador booleano do tipo "AND". As combinações incluídas foram: old age AND falls AND physical therapy, nas bases de dados PubMed, PEDro e Cochrane. Conforme o Quadro 1.

Quadro 1 - Combinações e resultados de busca.

\begin{tabular}{|l|l|l|}
\hline Base de dados & Combinação & Total de artigos encontrados \\
\hline PUBMED & $\begin{array}{l}\text { old age AND falls AND } \\
\text { physical therapy }\end{array}$ & 236 \\
\hline PEDRO & $\begin{array}{l}\text { old age AND falls AND } \\
\text { physical therapy }\end{array}$ & 6 \\
\hline COCHRANE & $\begin{array}{l}\text { old age AND falls AND } \\
\text { physical therapy }\end{array}$ & 103 \\
\hline
\end{tabular}

Fonte: Autores.

Foram definidos como critérios de inclusão ensaios clínicos randomizados no idioma em inglês. E foi decidido como critério de exclusão publicações de periódicos duplicados, periódicos não disponíveis e periódicos que não estivessem dentro do período dos últimos cinco anos (2016-2021).

De modo a identificar a abordagem dos artigos selecionados, a pesquisa desenvolveu-se a partir da juntura de 2 etapas de avaliação. A primeira etapa é nomeada de crivo inicial, e a segunda etapa nomeada de crivo final. 
No crivo inicial foi realizado uma leitura de título, palavras - chave, resumo, introdução, objetivos, metodologia, resultados e conclusão, foram selecionados estudos que estivessem em concordância com a temática, porém, estudos que são apresentaram compatibilidade com a temática, assim como os requisitos mencionados anteriormente, foram excluídos.

Na sequência, foi desenvolvido o crivo final, onde foi utilizada a escala PEDro que é composta por onze critérios, sendo eles, o de elegibilidade, o de distribuição de sujeitos em grupos, o de o estudo ser considerado cego ou duplo-cego, randomização, mensuração de resultados e medidas estatísticas. Com nota de 0 à 10 , o objetivo da escala é ajudar as pessoas que utilizam da base de dados a identificar quais os estudos que se encontram arquivados nas bases de dados. Além disso, a escala proporciona que seja identificado o grau de serventia da temática clínica e permite explorar informações estatísticas suficientes para a interpretação de resultados. Nessa etapa de avaliação estudos que apresentaram nota menor que 5 foram excluídos.

No decorrer da análise dos artigos foram revelados um total de 236 periódicos na base de dados PubMed, após a implementação dos critérios de exclusão foram excluídos 198 estudos, restando assim 38 estudos, em seguida foi implementado os critérios de inclusão onde foi excluído um total de 34 periódicos, restando assim 4 estudos. Subsequentemente, na base de dados PEDro foi revelado um total de 6 estudos que, após implementado os critérios de exclusão foram excluídos 2 estudos, restando 4 estudos. Quanto à base de dados Cochrane, foram revelados um total de 103 periódicos, contudo, após a implementação dos critérios de exclusão, foram excluídos 92 estudos, restando 11 estudos.

Posteriormente, os estudos restantes passaram por as etapas de avaliação que já foram mencionadas acima, na primeira etapa foram excluídos 2 artigos da base de dados PEDro, sendo 2 artigos excluídos por não obedecerem a temática, restando assim 2 artigos que foram selecionados para a segunda etapa avaliativa. Da base de dados PubMed, foram excluídos ainda 3 artigos por motivo de não obedecerem a temática, restando assim, 1 artigo que foi selecionado para a segunda etapa de avaliação. E da base de dados Cochrane foram excluídos 8 artigos por não obedecerem a temática, restando assim 3 estudos que foram selecionados para a segunda etapa. Na segunda etapa foi excluído 1 artigo da base de dados PEDRO por não alcançar o score. Quanto a base de dados PubMed e Cochrane, não houve exclusão de estudos, devido a terem conseguido nota a partir de 5 de acordo com a escala PEDro. Totalizando 5 ensaios clínicos presentes neste estudo. Conforme o fluxograma abaixo. 
Figura 1 - Fluxograma.

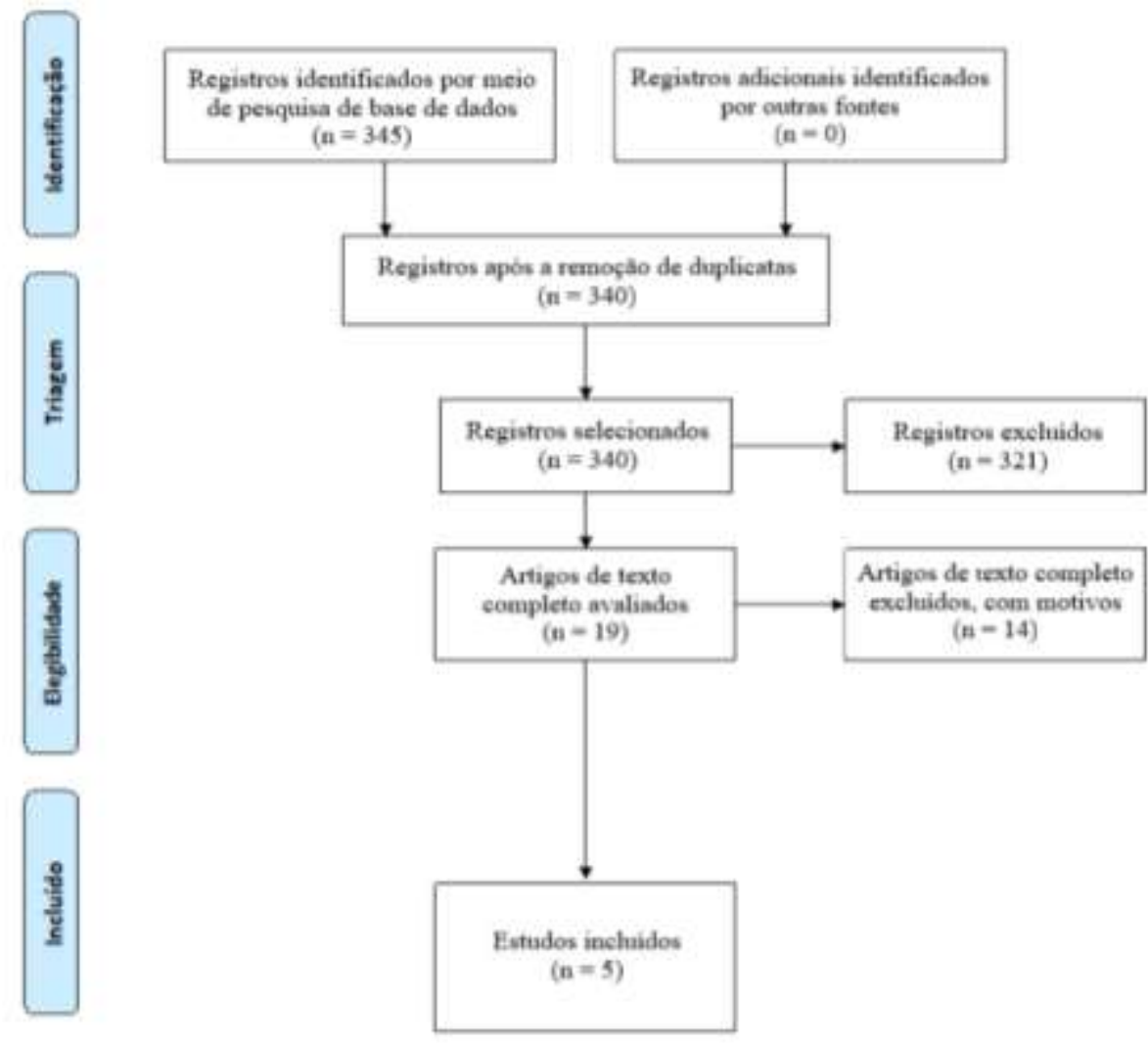

Fonte: Prisma (2008)

\section{Resultados e Discussão}

Segue abaixo a disposição dos artigos de acordo com autores, título, ano de publicação, objetivo geral e principais considerações:

Tabela 1 - Esquema das publicações científicas.

\begin{tabular}{l} 
Artigo 01 \\
\hline AUTOR (ES): BERGLAND, A.; LEVEILLE, G. S.; CEDERBOM, S. \\
TÍTULO: Efeitos de uma intervenção de medicina comportamental na dor, saúde e comportamento entre idosos residentes na \\
comunidade: um ensaio clínico randomizado \\
ANO DE PUBLICAÇÃO: 2019 \\
APLICAÇÃO DA ESCALA PEDro: 7,0 pontos \\
OBJETIVO GERAL: avaliar os efeitos de uma intervenção, com base em uma abordagem de medicina comportamental em \\
fisioterapia (BMPI), na incapacidade relacionada à dor e desempenho físico, bem como na intensidade da dor, catastrofização \\
da dor, níveis de atividade física, quedas eficácia e qualidade de vida relacionada à saúde (QVRS), comparando os efeitos ao \\
tratamento padrão. \\
PRINCIPAIS CONSIDERAÇÕES: A intervenção, baseada em uma abordagem de medicina comportamental, comparada \\
ao tratamento usual, teve um efeito positivo na incapacidade relacionada à dor, intensidade da dor, nível de atividade física, \\
QVRS, gerenciamento da vida cotidiana e autoeficácia. O BMPI pode ser uma intervenção baseada em evidências adequada \\
para idosos que vivem na comunidade, mesmo para aqueles que são muito idosos e frágeis. O BMPI pode apoiar e promover \\
o envelhecimento ativo e "envelhecer" da população-alvo, que é atualmente o principal objetivo de todas as intervenções \\
neste domínio.
\end{tabular}




\begin{abstract}
Artigo 02
AUTOR (ES): PÉREZ-SANTOS. S.; DELGADO-LIROLA. A.; GARCÍA-FARALDO. A.; ANDRADE-SÁNCHEZVAAMONDE. I.; VALEIRAS-RIO-DEL. M.; IZQUIERDO-ROSSI. M.; VARELA-SOTO. A.

TÍTULO: Reabilitação Vestibular com Sistema Posturográfico em Pacientes Idosos com Instabilidade Postural: É possível reduzir o número de sessões?

ANO DE PUBLICAÇÃO: 2020

APLICAÇÃO DA ESCALA PEDro: 7,0 pontos

OBJETIVO GERAL: avaliar se dois diferentes protocolos de RV com posturografia, um deles mais longo (dez sessões) e outro mais curto (cinco sessões), apresentam diferenças significativas na melhora do equilíbrio em pacientes idosos com instabilidade.

PRINCIPAIS CONSIDERAÇÕES: Os dois grupos de tratamento (20 pacientes por grupo) eram comparáveis em idade, sexo e avaliação do equilíbrio pré-RV. Em ambos os grupos, observamos uma melhora significativa no equilíbrio global (composto) após VR (49 $\pm 11,34$ vs $57 \pm 13,48, \mathrm{p}=0,007$, no grupo submetido a 10 sessões; $51 \pm 12,55$ vs $60 \pm 12,99$, p = 0,002,5 sessões). Em ambos os grupos, também observamos melhorias em outros parâmetros posturográficos (no SOT e nos limites de estabilidade), mas não nos escores timed up and go ou nos questionários. A comparação do nível de melhora alcançado em ambos os grupos não revelou diferenças significativas entre eles. Os protocolos de reabilitação vestibular por posturografia de 5 sessões em idosos com instabilidade postural são tão eficazes quanto os de 10 sessões para melhorar o equilíbrio em idosos com instabilidade postural.
\end{abstract}

\title{
Artigo 03
}

AUTOR (ES): SIEGRIST, M.; FREIBERGER, E.; GEILHOF, B.; SALB, J.; HENTSEHKE, C.; LANDENDOERFER, P.; LINDE, K.; HALLE, M.; BLANK, A. W.

TÍTULO: Prevenção de quedas em um ambiente de atenção primária

Os efeitos de uma intervenção de exercício complexo direcionado em um estudo randomizado de cluster

\section{ANO DE PUBLICAÇÃO: 2016}

APLICAÇÃO DA ESCALA PEDro: 5,0 pontos

OBJETIVO GERAL: investigar se a implementação de um programa de prevenção de quedas baseado em exercícios no ambiente alemão de cuidados primários (médicos de clínica geral), consistindo em 16 semanas de exercícios em grupo em combinação com uma casa individualizada, o programa de treinamento baseado em programas pode reduzir significativamente o número de quedas por indivíduo em idosos residentes na comunidade com alto risco de quedas em comparação com aqueles que recebem cuidados habituais por um período de um ano.

PRINCIPAIS CONSIDERAÇÕES: 40 clínicas gerais participaram do estudo. Sete práticas gerais desistiram após a randomização e antes de recrutar pacientes. Em 33 práticas, um total de 378 pacientes $(65$ a 94 anos, idade média 78,1 $\pm 5,9$ anos, 285 mulheres) foram incluídos. O tempo de recrutamento foi de julho de 2009 a março de 2010. Os motivos para a perda de acompanhamento ( $\mathrm{n}=78$ pacientes) foram documentados.

O programa de prevenção de quedas foi organizado em cooperação com as práticas gerais participantes no ambiente de vida dos pacientes. Em cada grupo de treinamento participaram de quatro a 12 participantes. No total, 181 dos pacientes (82\%) participaram de mais de dez sessões de treinamento. 46\% dos pacientes (101 de 222 participantes) realizaram o programa de exercícios em casa dez vezes ou mais (média 6,7 vezes). Nenhum efeito adverso foi observado durante o programa de exercícios (figura 1 )

Mais de 50\% de todos os pacientes relataram pelo menos uma queda no ano anterior ao estudo. Um programa complexo de prevenção de quedas em um ambiente de atenção primária foi eficaz na redução de quedas e lesões relacionadas a quedas em idosos residentes na comunidade em risco.

\section{Artigo 04}

AUTOR (ES): COVILL, L. G.; UTLEY, C.; HOCHSTEIN, C.

TÍTULO: Comparação de Ai Chi e terapia aquática baseada em deficiência para adultos mais velhos com problemas de equilíbrio: um estudo clínico

ANO DE PUBLICAÇÃO: 2017

APLICAÇÃO DA ESCALA PEDro: 6,0 pontos

OBJETIVO GERAL: determinar se Ai Chi fornece mais bem resultados do que a terapia aquática convencional baseada em deficiência (IBAT) para adultos mais velhos com déficits de equilíbrio.

PRINCIPAIS CONSIDERAÇÕES: Uma análise de modelo misto bidirecional (grupo por tempo) de covariância com pontuações de resultados iniciais como uma covariável não revelou nenhuma diferença entre os grupos em qualquer uma das medidas de resultados (BBS, $\mathrm{P}=0,53$; TUG, $\mathrm{P}=0,39 ; \mathrm{ABC}, \mathrm{P}=0,63$; NPRS, $\mathrm{P}=0,27$ ). A análise de medidas repetidas e os testes $t$ dependentes mostraram melhorias significativas no BBS $(\mathrm{P}=.00)$ e TUG $(\mathrm{P}=.03)$ após o APT. O ABC e NPRS não melhoraram significativamente $(A B C, P=0,27$; NPRS, $P=0,77)$. Não foram encontradas diferenças significativas nas medidas de equilíbrio, confiança no equilíbrio ou níveis de dor para idosos residentes na comunidade entre os programas Ai Chi e IBAT. As medidas de resultados físicos melhoraram com APT, mas as medidas relatadas pelo paciente não. Estudos 


\section{Artigo 05}

AUTOR (ES): SIPILÄ, S.; TIRKKONEN, A.; HÄNNINEN, T.; LAUKKANEN, P.; ALEN, M.; FIELDING, A. R.; KIVIPELTO, M.; KOKKO, K.; KULMALA, J.; RANTANEN, T.; SIHVONEN E. S.; SILLANPÄ̈̈, E.; NEELY, S. A.; TÖRMÄKANGAS, T.

TÍTULO: Promovendo caminhada segura entre idosos: os efeitos de uma intervenção de treinamento físico e cognitivo vs. treinamento físico isolado na mobilidade e quedas entre homens e mulheres idosos que vivem na comunidade (o estudo PASSWORD): desenho e métodos de um ensaio clínico randomizado

ANO DE PUBLICAÇÃO: 2018

\section{APLICAÇÃO DA ESCALA PEDro: 7,0 pontos}

OBJETIVO GERAL: determinar se uma combinação de treinamento físico e cognitivo (PTCT) tem maiores efeitos na velocidade de caminhada, custo de dupla tarefa na velocidade de caminhada e funções executivas em comparação com o treinamento físico $(\mathrm{PT})$ sozinho.

PRINCIPAIS CONSIDERAÇÕES: O estudo foi projetado para capturar os efeitos aditivos e possíveis efeitos sinérgicos do treinamento físico e cognitivo. Em conclusão, as evidências sobre os efeitos das intervenções de treinamento físico na velocidade de caminhada, prevenção de quedas e cognição entre residentes na comunidade, no máximo, idosos moderadamente ativos são contraditórias. Portanto, pesquisas que investiguem novas estratégias para promover uma caminhada segura em populações idosas são necessárias.

Fonte: Autores.

De acordo com Cederbom et al. (2019), o estudo teve como objetivo avaliar em idosos que residem sozinhos na comunidade os efeitos de uma abordagem da medicina comportamental em fisioterapia, a fim de analisar os seguintes itens: 1) se a dor incapacita a função física e 2) quanto à intensidade e convicção da dor, e o grau de exercício físico, queda da funcionalidade e qualidade de vida quando comparado à um tratamento convencional. Participaram do estudo, 105 pessoas a partir de 75 anos que sofresse de dores crônicas há no mínimo 3 meses e que morassem sozinhos. As 105 pessoas foram divididas em grupos denominados de grupo de intervenção (GI) com total de 52 participantes, e grupo de controle (GC) com 53 participantes.

O acompanhamento ocorreu por 12 semanas. Os dois grupos receberam orientações das atividades físicas, bem como dos benefícios da prática dos exercícios para o alívio de dores crônicas. Para o GI foram realizadas 9 visitas do fisioterapeuta para realizar as sessões e uma ligação para prestar assistência, cada visita dos participantes do GI em torno 30 e 45 minutos. Primeiramente, o fisioterapeuta realizou para o GI Análise Funcional do Comportamento (ANF) a fim de identificar se o indivíduo consegue realizar as atividades do dia a dia. Posteriormente foi passado exercícios funcionais de acordo com os achados da ANF. Os exercícios respeitaram à capacidade de sustentação de cada participante do GI e os exercícios foram aumentando gradativamente respeitando a funcionalidade e o estado de saúde de cada participante.

Já o GC recebeu o tratamento convencional que abrangia as orientações que foram mencionadas anteriormente quanto à recomendação da prática de atividade física e no lugar das visitas, o GC recebeu chamadas de telefone com duração de 20 minutos cada ligação. No decorrer do estudo houve desistências restando, 43 participantes no GI e 41 no GC. Os resultados achados para o item 1 foram: Houve diferença considerável na diminuição da incapacidade da dor entre o GI e GC. E para o item 2 foram: Houve diferença considerável examinada para a diminuição da intensidade da dor e aumento considerável do componente físico, mental, e aumento da funcionalidade e qualidade de vida entre o GI e GC.

Os resultados do estudo mostraram que os indivíduos do GI tiveram diminuição de quedas e tiveram melhora da funcionalidade quando comparado aos participantes do GC. Os integrantes do GI também tiveram como resultados diminuição da ingestão de remédios para dor, que também é fator importante para redução de risco de queda, pois algumas medicações causam efeitos colaterais que deixam os idosos mais vulneráveis a cair. Além disso, os participantes do GI relataram que 
continuaram com os exercícios em comparação com o GC. Dessa forma o autor conclui que uma abordagem da medicina comportamental em fisioterapia diminui a incapacidade relacionada à dor e à intensidade da dor, e é adequado para serem feitos de forma domiciliar, além de melhorar o grau de atividade física, aumento da funcionalidade, diminuindo o risco de quedas em idosos, além de promover maior qualidade de vida para a população idosa por gerar um envelhecimento ativo.

Entretanto, o estudo desenvolvido por Soto-Varela et al (2020), teve como objetivo analisar se a reabilitação com uso de sistema de posturografia apresenta o mesmo nível de eficácia para melhora do equilíbrio e redução do número de quedas quando comparado um tratamento de 10 sessões com um tratamento com 5 sessões. Participaram do estudo 40 pessoas com idade a partir de 65 anos que apresentassem risco elevado de quedas. Os participantes foram divididos em dois grupos, sendo o primeiro com 20 participantes que seguiram um protocolo de 10 sessões, e o segundo com 20 participantes que seguiram um protocolo de 5 sessões. Sobre o grupo que seguiu o protocolo de 10 sessões, a reabilitação consistiu em duas intervenções distintas, onde 9 pacientes fizeram a reabilitação pelo programa Smart Equitest e 11 pacientes fizeram a reabilitação com o dispositivo móvel Vertiguard. Para o programa Smart Equitest foi utilizado um protocolo onde a cada sessão eram feitos 10 exercícios que foram adaptados respeitando a individualidade de cada paciente.

Os exercícios continham biofeedback visual com acompanhamento do movimento em tempo real. Em determinados exercícios os participantes mantinham seu centro de gravidade (COG) sobre a base do suporte, já em outros momentos o COG era deslocado para alvos específicos, já que também a base que oferece o suporte também é capaz de mover-se para responder o movimento do paciente. A dificuldade do exercício foi aumentada gradativamente e a sessão durava em torno de 15 minutos. Diferente do Smart Equitest, o dispositivo móvel Vertiguard, funciona com uma bateria fixa num cinto que é colocado no quadril do paciente e possui um estimulador de vibrações para os respectivos lados: anterior, posterior, esquerdo e direito. Nele, foram realizadas 6 tarefas, onde em cada sessão, foram feitas 5 repetições dessas 6 tarefas que duraram 20 segundos cada repetição.

O paciente recebia um estímulo vibro tátil que mostravam a oscilação corporal maior do que as oscilações que já haviam sido definidas anteriormente. Dessa forma quanto mais a oscilação corporal fugisse do que já havia sido definido de parâmetro anteriormente, maior era a vibração que o paciente sentia, para que ele pudesse perceber e fazer as correções. A duração de cada sessão foi em torno de 15 minutos e a dificuldade dos exercícios foi aumentada de forma gradativa. E sobre o grupo que seguiu o protocolo de 5 sessões também foram utilizadas as mesmas intervenções que as grupo que seguiu protocolo de 10 sessões utilizou. Do grupo, 10 pacientes fizeram a reabilitação com uso do programa Smart Equitest e 10 paciente fizeram a reabilitação com uso do dispositivo móvel Vertiguard. As sessões ocorreram em duas semanas sendo realizadas em dia sim, dia não. Os resultados mostraram que o sistema de posturografia melhorou o equilíbrio global dos participantes e quanto aos grupos, não houve diferença significativa no grupo que seguiu o protocolo de 10 sessões quando comparado ao grupo que seguiu protocolo de 5 sessões. Dessa forma, o autor concluiu que, a reabilitação com sistema de posturografia melhorou o equilíbrio dos participantes, o que contribui para a redução do risco de quedas, e que protocolos de 5 sessões são tão eficazes como protocolos de 10 sessões, o que ajuda na redução de custos para realização do tratamento.

Contudo, Siegrist et al (2016), realizou um estudo, cujo objetivo primário foi averiguar se um programa de exercícios em grupo quando comparado à cuidados habituais possui mais eficácia no quesito redução do risco de quedas. E como objetivo secundários, os autores buscaram averiguar a ocorrência de lesão por queda e os efeitos do programa de exercícios na função física e no medo de cair. Participaram do estudo 378 pessoas com idade a partir de 65 anos com risco elevado de cair. Os participantes foram divididos em grupos denominados de Grupo de Intervenção (GI) com 222 pessoas e Grupo de Controle (GC) com 156 pessoas. O GI realizou um programa de exercícios que foi trabalhado no decorrer de 16 semanas, sendo realizado 1 hora de treinamento/semana, onde foi trabalhado treino de marcha, equilíbrio, força e potência. Quanto ao GC, não recebeu um tratamento organizado pois, faltou orientação por parte dos clínicos gerais. O que foi observado foi que durante os 
12 meses em que a pesquisa foi realizada houve um número de 291 quedas no GI, enquanto 129 participantes não relataram ter sofrido quedas e no GC, 367 quedas ocorreram, enquanto 79 participantes não relataram quedas. No quesito lesão por queda, foram realizadas 479 entrevistas por ligação telefônica onde foi ofertado informações sobre lesão por queda.

No GI, 63 de 222 participantes relataram uma lesão por queda, quanto que no GC, 59 de 156 participantes relataram uma lesão relacionada à queda. Dessa forma ficou nítido que a taxa de ocorrência de lesões por conta de queda foi menor no GI. Respondendo aos objetivos secundários foi realizado o Timed-Up-and-Go onde o GI apresentou melhorias e o GC necessitou de mais tempo para realizar o teste. No Teste de Levantamento de Cadeira não houve diferença significativa no tempo entre GI e GC. No teste de mRomberg o GI apresentou melhor desempenho no equilíbrio quando comparado ao GC. E na Escala de Eficácia de Quedas (FES-I) o medo de cair foi menor no GI do que no GC. Dessa forma fica explicito que um programa de exercícios para prevenção de quedas em idosos pode diminuir significativamente o risco de cair e consequentemente também reduz o risco de lesão quando comparado aos que recebem cuidados habituais. Além disso o medo de cair reduziu de forma considerável e na função física foram observados efeitos positivos. Dessa forma, o autor conclui que a utilização de uma clínica geral é o lugar ideal para realizar um programa de exercícios para prevenção de quedas.

Na sequência, Covill et al. (2017) desenvolveram um estudo que teve como objetivo analisar se a terapia aquática Ai Chi comparado com a terapia aquática tradicional baseada em evidência possui mais bem resultados na melhoria do equilíbrio, diminuição da dor, diminuição do risco de queda e desenvolvimento de confiança no equilíbrio. Participaram do estudo 48 pessoas com idade de 65 à 85 anos de idade que relataram medo de cair, que caíram no último ano e/ou foram encaminhados pelo médico treino de equilíbrio. Os participantes foram divididos em dois grupos sendo o grupo Ai Chi com 22 participantes e o grupo de terapia aquática tradicional baseada em evidência (IBAT) com 26 participantes. O grupo Ai Chi seguiu o seguinte protocolo: 1. Aquecimento por 5 à 10 minutos que incluiu andar para os lados, para frente e para trás e andar sob os calcanhares e ponta dos pés; 2 .

Foram realizados exercícios preparatórios para o Ai Chi como agachamento, respiração diafragmática, facilitação transversa do abdômen e postura com facilitação transversa do abdômen; 3. Foi realizado 20 minutos de exercício que seguiram a sequência Ai Chi, respeitando a individualidade de cada participante; 4. Exercícios que promoveram relaxamento e que trabalharam a flexibilidade de MMSS e MMII. Quanto ao grupo IBAT, o protocolo que foi seguido consistiu em: 1. Caminhada de 5 à 10 minutos para aquecer; 2. Sessão de 20 minutos onde foi feito treino de equilíbrio também respeitando a individualidade de cada participante; 3 . Exercícios que promoveram relaxamento e 5 minutos de exercício para ganho de flexibilidade de MMSS e MMII. No decorrer do estudo 16 pessoas desistiram, restando 32 pessoas que concluíram. Dessa forma, os resultados e a conclusão que seu foi que não houve diferença significativa entre Ai Chi e IBAT para diminuição do risco de quedas e os autores concluíram que se faz necessário mais estudos acerca do assunto.

Assim sendo, Sipilä et al (2018), realizou um estudo que teve como objetivo avaliar se o treino físico + treino cognitivo era capaz me melhorar a velocidade da marcha quando comparado com o treino físico de forma isolada. A intervenção foi realizada no decorrer de 1 ano e participaram do estudo 310 pessoas com idade de 75 à 85 anos, que foram divididos em dois grupos iguais, grupo de Treinamento Físico + Cógnito com 155 pessoas e o grupo de Treinamento Físico Isolado também com 155 pessoas participando. Foram realizados treinos com supervisão e exercícios em casa. A intervenção foi iniciada com um seminário de introdução afim de expor os benefícios da prática da atividade física em idosos. Foi fornecido instruções e um cronograma para as sessões supervisionadas e individuais, além disso, também foram dadas as orientações para o treino cognitivo, ambos os grupos participaram do seminário.

As intervenções consistiram em sessões semanais em grupos de 10 à 15 participantes. Para os exercícios em casa foi mantido um diário onde havia todos os registros dos exercícios realizados. Para facilitar a aceitação das intervenções, nos dois primeiros meses foi feito apenas o treino físico para os dois grupos. O treino físico consistiu em: exercícios aeróbicos e treino 
de resistência e equilíbrio. O exercício aeróbico consistiu na realização de sessões de caminha ao ar livre em uma pista circular de 400m por 10 à 20 minutos. Já o treino de resistência foi realizado em 3 academias, os exercícios utilizados foram: leg press, leg curl, extensão de MMII, abdução e adução de quadril, extensão de quadril, panturrilha, exercício de remo, supinação torácica e extensão de cotovelo. Os programas de exercício em casa incluíram fortalecimento de MMII, treino de equilíbrio e alongamento dos grupos musculares básicos. Quanto ao treino cognitivo, foi realizado por meio de computador onde foram realizadas 4 tarefas que foram divididas em dois blocos. Nesses blocos foram trabalhados a memória, tarefa de cores, tarefa de letras e tarefa de números e a sessão durou 20 minutos. Os resultados achados mostraram que as evidências foram contraditórias, alguns participantes tiveram benefícios com o treinamento físico e não teve com o treino cognitivo, enquanto outros já tiveram benefícios com o treino cognitivo e não teve com treinamento físico. Dessa forma os autores concluem que se faz necessário novas estratégias acerca do assunto.

\section{Considerações Finais}

O estudo realizado teve como finalidade realizar um levantamento na literatura para identificar as abordagens de fisioterapia que apresentassem melhor resultados na prevenção de quedas em idosos. Sendo assim, ficou claro, que protocolos de programas de exercício físico e sistema de posturografia propiciam melhor resultados para a prevenir e diminuir o risco de cair, além de promover outros benefícios como diminuição do medo de cair, aumento da funcionalidade e qualidade de vida da pessoa idosa. Contudo, a terapia com sistema de posturografia mostrou que tanto faz 10 sessões como 5 sessões, os resultados ainda se mostram eficazes, reduzindo assim custos, uma vez que a terapia por sistema de posturografia pode ser um desafio no sentido socioeconômico.

Por conseguinte, é importante destacar a importância de que mais estudos relacionados a temática sejam realizados, a fim de contribuir para uma melhor escolha por parte do fisioterapeuta ao escolher condutas que visem trabalhar a prevenção de quedas em idosos.

\section{Referências}

Baets, A. (1986). O deficiente físico e a pessoa idosa. Servir (Lisbon, Portugal), 34(5-6), 261-263.

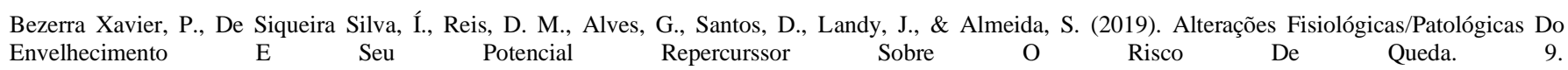
https://editorarealize.com.br/revistas/cieh/trabalhos/TRABALHO_EV125_MD1_SA2_ID198_29042019233637.pdf

Cederbom, S., Leveille, S. G., \& Bergland, A. (2019). Effects of a behavioral medicine intervention on pain, health, and behavior among communitydwelling older adults: A randomized controlled trial. Clinical Interventions in Aging, 14, 1207-1220. https://doi.org/10.2147/CIA.S208102

Chittrakul, J., Siviroj, P., Sungkarat, S., \& Sapbamrer, R. (2020). Multi-System Physical Exercise Intervention for Fall Prevention and Quality of Life in PreFrail Older Adults: A Randomized Controlled Trial. International journal of environmental research and public health, $17(9), 3102$.

Covill, L. G., Utley, C., \& Hochstein, C. (2017). Comparison of Ai Chi and Impairment-Based Aquatic Therapy for Older Adults with Balance Problems: A Clinical Study. Journal of Geriatric Physical Therapy, 40(4), 204-213. https://doi.org/10.1519/JPT.0000000000000100

Lach, H. W., \& Noimontree, W. (2018). Fall Prevention Among Community-Dwelling Older Adults: Current Guidelines and Older Adult Responses. Journal of gerontological nursing, 44(9), 21-29.

Lipardo, D. S., \& Tsang, W. (2018). Falls prevention through physical and cognitive training (falls PACT) in older adults with mild cognitive impairment: a randomized controlled trial protocol. BMC geriatrics, $18(1), 193$.

Liu-Ambrose, T., Davis, J. C., Best, J. R., Dian, L., Madden, K., Cook, W., Hsu, C. L., \& Khan, K. M. (2019). Effect of a Home-Based Exercise Program on Subsequent Falls Among Community-Dwelling High-Risk Older Adults After a Fall: A Randomized Clinical Trial. JAMA, 321(21), 2092-2100.

Lopes, D. de F., Santos, S. D. dos, Souza, S. A. N. de, Silva, E. G. da, \& Santos, W. L. dos. (2019). Fatores relacionados a quedas em idosos. Revista de Iniciação Científica e Extensão, 2(3), 131-138.

Lusardi, M. M., Fritz, S., Middleton, A., Allison, L., Wingood, M., Phillips, E., Criss, M., Verma, S., Osborne, J., \& Chui, K. K. (2017). Determining Risk of Falls in Community Dwelling Older Adults: A Systematic Review and Meta-analysis Using Posttest Probability. Journal of geriatric physical therapy (2001), 40(1), 1-36. 
Research, Society and Development, v. 11, n. 1, e33311124938, 2022

(CC BY 4.0) | ISSN 2525-3409 | DOI: http://dx.doi.org/10.33448/rsd-v11i1.24938

Martins, F., Cristina, R., Silveira, G., \& Mundim, M. M. (2021). A importância da fisioterapia na prevenção de quedas em idosos - artigo de revisão The importance of physiotherapy in the prevention of falls in the elderly - review article. 30

Morello, R. T., Soh, S. E., Behm, K., Egan, A., Ayton, D., Hill, K., Flicker, L., Etherton-Beer, C. D., Arendts, G., Waldron, N., Redfern, J., Haines, T., Lowthian, J., Nyman, S. R., Cameron, P., Fairhall, N., \& Barker, A. L. (2019). Multifactorial falls prevention programmes for older adults presenting to the emergency department with a fall: systematic review and meta-analysis. Injury prevention : journal of the International Society for Child and Adolescent Injury Prevention, 25(6), 557-564.

Park S. H. (2018). Tools for assessing fall risk in the elderly: a systematic review and meta-analysis. Aging clinical and experimental research, $30(1), 1-16$.

Phelan, E. A., \& Ritchey, K. (2018). Fall Prevention in Community-Dwelling Older Adults. Annals of internal medicine, 169(11), ITC81-ITC96.

Sherrington, C., Michaleff, Z. A., Fairhall, N., Paul, S. S., Tiedemann, A., Whitney, J., Cumming, R. G., Herbert, R. D., Close, J., \& Lord, S. R. (2017). Exercise to prevent falls in older adults: an updated systematic review and meta-analysis. British journal of sports medicine, 51(24), $1750-1758$.

Siegrist, M., Freiberger, E., Geilhof, B., Salb, J., Hentschke, C., Landendoerfer, P., Linde, K., Halle, M., \& Blank, W. A. (2016). Originalarbeit: Sturzprävention in Hausarztpraxen: Effekte eines komplexen Trainingsprogramms im Rahmen einer cluster-randomisierten Studie. Deutsches Arzteblatt International, 113(21), 365-372. https://doi.org/10.3238/arztebl.2016.0365

Sipilä, S., Tirkkonen, A., Hänninen, T., Laukkanen, P., Alen, M., Fielding, R. A., Kivipelto, M., Kokko, K., Kulmala, J., Rantanen, T., Sihvonen, S. E., Sillanpää, E., Stigsdotter-Neely, A., \& Törmäkangas, T. (2018). Promoting safe walking among older people: The effects of a physical and cognitive training intervention vs. physical training alone on mobility and falls among older community-dwelling men and women (the PASSWORD study): Design and methods of a randomized controlled trial. BMC Geriatrics, 18(1), 1-12. https://doi.org/10.1186/s12877-018-0906-0

Soto-Varela, A., Rossi-Izquierdo, M., Del-Río-valeiras, M., Vaamonde-Sánchez-andrade, I., Faraldo-García, A., Lirola-Delgado, A., \& Santos-Pérez, S. (2020). Vestibular rehabilitation using posturographic system in elderly patients with postural instability: Can the number of sessions be reduced? Clinical Interventions in Aging, 15, 991-1001. https://doi.org/10.2147/CIA.S263302

Souza, L. H. R., Brandão, J. C. da S., Fernandes, A. K. C., \& Cardoso, B. L. C. (2017). Queda Em Idosos E Fatores De Risco Associados. Revista Brasileira Ciências Da Saúde - USCS, 15(54), 55-60. https://doi.org/10.13037/ras.vol15n54.4804

Zhang, W., Low, L. F., Schwenk, M., Mills, N., Gwynn, J. D., \& Clemson, L. (2019). Review of Gait, Cognition, and Fall Risks with Implications for Fall Prevention in Older Adults with Dementia. Dementia and geriatric cognitive disorders, 48(1-2), 17-29. 